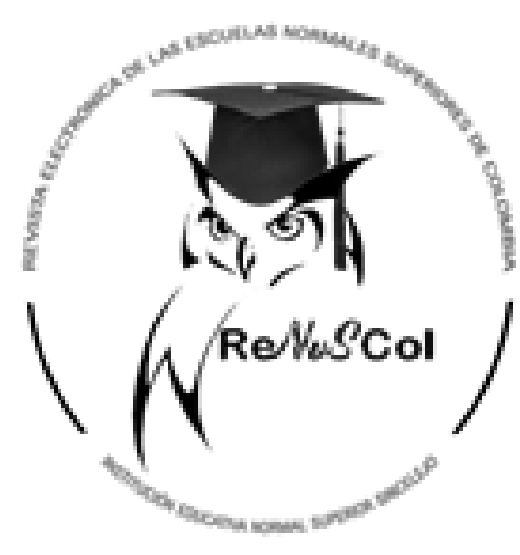

Cómo citar este texto:
Mag. Jorge Velasco Lara

Email: georgealejandria1@gmail.com

ORCID: 0000-0002-2341-0702

Docente e Investigador de la Normal Superior Sincelejo.

Esp. Beatriz Torres Balmaceda

Email: beatob596@gmail.com

\section{ORCID: 0000-0003-3233-813X}

Docente e Investigador de la Normal Superior Sincelejo.

Mag. Neiza Ramos Echeverría

Email: Nerrachi@yahoo.es

ORCID: 0000-0001-9379-8338

Docente e Investigador de la Normal Superior Sincelejo

Esp. Néver Suarez Contreras

Email: neversu08@gmail.com

\section{ORCID: 0000-0003-0062-1897}

Docente e Investigador de la Normal Superior Sincelejo.

Mag. Katherin González Casilla

Email: keytgon_22@ @otmail.com

ORCID: 0000-0001-5104-2372

Docente e Investigador de la Normal Superior Sincelejo.

Lic. Jader Díaz Conde

Email: jaderdiaz1988@gmail.com

ORCID: 0000-0001-9855-8317

Docente e Investigador de la Normal Superior Sincelejo.

Velasco L. J., Torres. B., Ramos, E. N., Suárez C. N., González C. K., Díaz C. J. (2021). Incidencia del pensamiento crítico y las habilidades sociocognitivas en los procesos teórico prácticos de estudiantes del Programa de Formación Complementaria. ReNoSCol. No. I, Vol I. Enero-Junio/ 2021. Pp. 41-57. Institución Educativa Normal Superior Sincelejo. URL disponible en: http://www.eumed.net/rev/renoscol.html

Recibido: noviembre 2020 . Aceptado: abril de 2021. Publicado: junio de 2021.

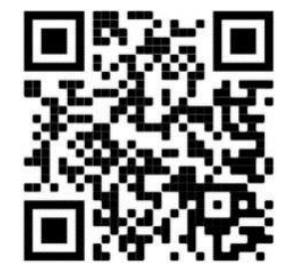


Título: Incidencia del pensamiento crítico y las habilidades sociocognitivas en los procesos teórico prácticos de estudiantes delPrograma de Formación Complementaria.

Resumen: Dado el propósito que caracteriza este trabajo investigativo desde la incidencia que presentan las habilidades de pensamiento crítico y habilidades sociocognitivas como una apuesta en la mejora de los procesos teórico-prácticos de los docentes en formación inicial del programa de formación complementaria de la Institución Educativa Normal Superior de Sincelejo, se parte de la identificación de estashabilidades ya señaladas desde un análisis mixto que en aplicabilidad secuencial de instrumentos cuantitativos y cualitativos y su posteriorlectura (spss y atlas-ti), constatara datos relevantes respecto a indicadores específicos de habilidades de pensamiento crítico y habilidades sociocognitivas, cuyo diagnóstico tomara relevancia como punto de partida para determinar una ruta metodológica que conciba la apropiación de estrategias problematizadoras en procesos de intervención pedagógica futura para la mejora en estas habilidades.

Palabras clave: Pensamiento Crítico, Habilidades de Pensamiento Crítico, Interacciones Sociocognitivas, Habilidades Sociocognitivas, Estrategias problematizadoras

Title: Incidence of the critical thought and the abilities socio-cognitive in students' theoretic practical processes ofFormation's Program Complementary.

Summary: Once the purpose that characterizes this investigating work from the incidence that the abilities of critical thought and abilities show sociocognitive like a bet in the improvement of the theoretic practical processes of the teachers in initial formation of the program of complementary formation of Sincelejo's Educational Normal Superior Institution, was given it breaks of the identification of these abilitiesright now indicated from a mixed analysis than in sequential applicability of quantitative and qualitative instruments and his later reading(spss and atlas you ), verify relevant data in relation to specific indicators of abilities of critical thought and abilities socio-cognitive, whose diagnosis take relevance like point of Departure to determine a route methodological that the appropriation of strategies conceive problematizadoras in processes of pedagogic future intervention for the improvement in these abilities.

Key words: Critical thought, Interactions Socio-cognitive, Skill's Socio-cognitive, Strategies, Critical thought, problem skill's.

Título: Incidência do pensamento crítico e as habilidades sociocognitivas nos processos teórico práticos de estudantes do Programa de Formação Complementar.

Resumo: Dado o propósito que caracteriza este trabalho inquiridor da incidência que apresentam as habilidades de pensamento crítico e habilidades sociocognitivas como uma aposta na melhora dos processos teórico-práticos dos docentes em formaçãoinicial do programa de formação complementar da Instituição Educativa Normal Superior do Sincelejo, parte-se da identificação destas habilidades já assinaladas de uma análise mista que em aplicabilidade seqüencial de instrumentos quantitativos e qualitativos e sua posterior leitura (spss e atlas-ti), constatasse dados relevantes respeito a indicadores específicos de habilidades de pensamento crítico e habilidades sociocognitivas, cujo diagnóstico tomasse relevância como ponto de partida para determinar uma rota metodológica que conceba a apropriação de estratégias problematizadoras em processos de intervenção pedagógica futura para a melhora nestas habilidades.

Palavras chave: Pensamento Crítico, Habilidades de Pensamento Crítico, Interações Sociocognitivas, Habilidades Sociocognitivas, Estratégias problematizadoras.

\section{INTRODUCCIÓN.}

La formación inicial de docentes es un factor importante para la educación, ya que de ésta depende el actuar del maestro en el aula, entre otros, como también es clave en la forma como se desenvuelve y adquiere las competencias el futuro maestro, al igual que el aprendizaje y como éste es entendido y asimilado por sus estudiantes. Sin olvidar que dicha interacción es clave en los procesos de enseñanza y aprendizaje, pues a través de esta, el conocimiento se construyeen espacios donde el docente pone en juego sus métodos y sus concepciones reflejadas en la forma como se orienta el acto pedagógico para generación y asimilación de los saberes.

Desde esta perspectiva, se espera que la educación al intervenir con sus métodos transforme la sociedad a partir de las aplicaciones pedagógicas presentes en el proceso de enseñanza y aprendizaje. En dicho accionar pedagógico,los estudiantes en formación adquieren los conocimientos y experiencias que posibilitan la formación de sujetos íntegros y autónomos, capaces de resolver 
las diferentes situaciones que se les presente en la vida cotidiana.

Los futuros maestros están expuestos a retos, dentro de ellos tenemos la observación, esta no debe ser simple sino crítica, poniendo especial atención a las actitudes, comportamientos, intereses, habilidades y otros aspectos de los estudiantes, para así poder detectar y atender las necesidades de cada uno de ellos, del mismo modo, la utilización de estrategias apropiadas para una buena enseñanza de acuerdo a las necesidades del contexto. En ese sentido, la sociedad demanda docentes competentes, con habilidades, actitudes acordes con las exigencias y retos de la sociedad actual y profesionales íntegros comprometidos con su labor. Todas estas características, se adquieren durante el proceso de formación inicial de docentes, y en la medida del ejercicio mismo, desde la adquisición de experiencias en los diferentes escenarios pedagógicos, donde se conjugan la teoría y la práctica.

En las practicas pedagógicas de los estudiantes se evidencia un accionar en el aula atendiendo a las directrices de un enfoque critico que sigue la Institución Educativa Normal Superior de Sincelejo (IENSS), desde ello, se ha tratado de implementar el quehacer pedagógico en el aula y pese a que existe desde el Proyecto Pedagógico e Investigativo (PPI) un direccionamiento para que el estudiante articule los distintos referentes teóricos del currículo como: los Derechos Básicos de Aprendizaje (DBA), estándares de competencias, didácticas específicas, Mallas de Aprendizaje, Matrices de Referencia, Orientaciones Pedagógicas, Competencias Transversales y Retos del Saber; en ocasiones se percibe que el estudiante cumple con llevar un formato en el plan de clases, sin embargo, la reflexión de dicho actuar pedagógico no se evidencia. Esto debido a la falta de fundamento y accionar pedagógico, lo que lleva a reflexionar sobre la falta de fundamentos teóricos que soporten la práctica del docente.

En otras circunstancias se encuentran estudiantes que si poseen los referentes teóricos del currículo y procuran en su quehacer transformar el contexto; sin embargo es entonces, donde sus docentes titulares de los escenarios de practica pedagógica presentan una resistencia originada en la carencia de dichos fundamentos, dificultándose con ello el desarrollo de experiencias innovadoras en procura de alcanzar ambientes de aprendizaje efectivos y por ende el consecuente desarrollo de habilidades de pensamiento crítico y de habilidades sociocognitivas en cada uno de los actores.

En ese sentido, el docente en formación inicial ha de reconstruir activamente los conceptos de la pedagogía, incorporándolos en sus estructuras de pensamiento y poniéndolos en ejecución en su quehacer, no obstante, para ello, debe considerar que, el aspecto pedagógico integra entonces, la conceptualización de la enseñanza a nivel teórico y de la investigación sobre ésta, como el conocimiento experiencial del maestro basado en la práctica de la docencia en el aula durante el desarrollo curricular. Estos dos niveles de la enseñanza -el teórico y el práctico- aportan conocimientos indispensables para la constitución del currículum y su ejecución, hecho que implica una acción reflexiva del estudiante que se da a la tarea de interactuar, discernir e integrar los conocimientos de un contexto para encarnarlos críticamente.

Ahora bien, teniendo en cuenta las anteriores percepciones desde un propósito que debe enmarcar al docente en formación inicial desde su practicidad en escenarios educativos donde incursionen para validar sus procesos teórico - prácticos, concibiendo habilidades de pensamiento crítico y habilidades sociocognitivas, este trabajo investigativo se fundamenta a partir de un análisis 
que permita visualizar niveles de desempeño respecto a las habilidades de pensamientocrítico y cómo estas influyen en las habilidades sociocognitivas. Finalmente, determina una secuencialidad didáctica para concebir estrategias problematizadoras que a partir de su posible aplicabilidad potencie estas habilidades en los docentes en formación inicial de la Institución Educativa Normal Superior de Sincelejo.

\section{Pensamiento Crítico desde Facione}

"Algunas veces nos hemos preguntado qué es exactamente pensamiento crítico y por qué se considera tan útil e importante... Usted no quisiera tener que memorizar una definición que apareciera en la página, ¿cierto? Eso sería una bobada, casi contraproducente. El objetivo aquí es ayudarle a agudizar sus habilidades de pensamiento crítico y a cultivar su espíritu de pensador crítico. Si bien la memorización definitivamente tiene muchos usos valiosos, fomentar el pensamiento crítico no es uno de ellos" (Facione, p. 2007, p. 2).

Estas consideraciones expuestas por Facione develan la complejidad de establecer un constructo teórico que conceptualice el pensamiento crítico, siendo esto un tema de discusión que puso a repensar la manera de enseñar y evaluar al pensamiento crítico, pero que llevó a un consenso de expertos (Fraser,Bailin, Epstein, Ennis; Paul, Facione, entre otros) que confluyeron en un concepto que lo definía como “Juicio auto-regulado", pero que se construye socioculturalmente (Vélez, 2013).

Desde esa óptica colectiva, Facione P. (1990), nos aclara que el Pensamiento Crítico:

Es el juicio auto-regulado y con propósito que da como resultado interpretación, análisis, evaluación e inferencia, como también la explicación de las consideraciones de evidencia, conceptuales, metodológicas, criteriológicas o contextuales en las cuales se basa ese juicio. El PC es fundamental como instrumento de investigación. Como tal, constituye una fuerza liberadora en la educación y un recurso poderoso en la vida personal y cívica de cada uno. Si bien no es sinónimo de buen pensamiento, el PC es un fenómeno humano penetrante, que permite auto-rectificar (p. 21).

Esta conceptualización dio paso para concebir dos dimensiones básicas que configuran al pensamiento crítico. Por un lado habilidades cognitivas en las que se pueden señalar la interpretación, análisis, evaluación, la inferencia, la explicación, y la auto-regulación; y la otra dimensión, está relacionada con las disposiciones o actitudes personales que caracterizan a una persona que piensa de manera crítica, al ser sistemática, buscadora de la verdad, analítica, reflexiva, de mente abierta y confiada en el razonamiento (Facione, 2007, p. 23). Dentro de estas habilidades de pensamiento crítico, existe una caracterización de criterios e indicadores que les diferencian y determinan además el proceso de complejidad de cada una de ellas. De manera textual, Facione (2007, pp. 4 - 6) conceptualiza cada una de ellas desde sus indicadores más relevantes que ser expondrán más adelante a lo largo de este trabajo investigativo.

\section{Interacciones y habilidades Sociocognitivas}

Las personas establecen relaciones de comunicación con sus semejantes, de manera que, es un ser social por naturaleza, y estas relaciones de interacción, representan una condición inherente a la resolución de sus necesidades desde las más básicas, hasta las más complejas y superiores (Farkas et al. 2006), y a las cuales se les puede referir como una manifestación de interacción 
social. Ahora bien, para dimensionar los acercamientos teóricos que se han construido respecto a las interacciones sociocognitivas, es necesario partir de su diferenciación con las interacciones sociales, de manera que, no se confunda el propósito de este trabajo investigativo.

En correspondencia a lo anterior, se puede decir que las interacciones sociales obedecen a conductas verbales y no verbales que dan lugar a interacciones con los demás en diferentes contextos sociales (Rinn \& Markle, 1979, citado en Farkas et al, 2006), no obstante sus manifestaciones son mediatizadas por factores cognitivos a las que se le denominan interacciones o habilidades sociocognitivas, donde se ven expuestos procesos cognitivo entre las distintas situaciones-estímulos de carácter interpersonal como la resolución de problemas, características del estilo atribucional, desde la causalidad de eventos o locus de control como a la atribución acerca de la intencionalidad de la conducta con los demás y la habilidad de tomar la perspectiva del otro (Shure \& Spivack, 1978).

Tratar de profundizar en el aspecto teórico de las habilidades e interacciones sociocognitivas, nos ha llevado a concebir que esta brida una perspectiva psicopedagógica del aprendizaje con gran potencial académico, sin embargo, aunque existan pocos trabajos investigativos que soporten su validez de manera reiterativa en las escuelas, ofrece aspectos determinantes como la motivación, la autorregulación del aprendizaje y la autoeficacia en procesos formativos de docentes (Chávez y Rodríguez, 2017).

De esta forma, se concibe esta teoría desde sus constructos y postulados en el propósito mismo que la caracteriza desde un proceso en el que:

El sujeto transforma sus habilidades mentales y creencias epistemológicas en habilidades concretas y conocimientos específicos. En este modelo teórico el individuo que aprende se percibe dentro de un ambiente cambiante que lo obliga a realizar constantes esfuerzos metacognitivos de evaluación y realimentación para ajustar sus conductas y estrategias; consecuentemente, el aprendizaje esvisto como una interacción entre elementos intrapersonales, conductuales y ambientales (Schunk y Zimmerman, 1994; Schunk, 2004; Azevedo, 2007; Zimmerman, 2008; Chávez y Rodríguez, 2017 , p. 49).

Para acercarnos más a la comprensión de la teoría sociocognitiva es necesario considerar el análisis del aprendizaje autorregulado, la autoeficacia y la motivación, de modo que, desde sus constructos teóricos, se entiende que "el sujeto puede ejercer cierto locus de control respecto a sus conductas y tareas, además de ello, enfatiza cómo las personas interactúan e influyen su ambiente, en lugar de ser pasivamente controladas" (Chávez y Rodríguez, 2017, p. 5), siendo esto un factor que influye en el aprendizaje autorregulado de los educandos, en el sentido que la percepción o creencia que estos tengan de su autoeficacia (autoconcepto) influye en la manera como pueden cumplir con sus propósitos; por lo que si su autoeficacia es elevada, menciona el autor (Bandura, 1976), la procura de llegar al éxito de sus propósitos se da de manera persistente, activa y motivada; $\sin$ embargo cuando este autoconcepto es bajo, el estudiante evita compromisos y su proximidad al fracaso y frustración es más 
latente.

Otro aspecto aquí mencionado son las habilidades sociocognitivas, las cuales presentan unos indicadores específicos, que si bien se han relacionado a lo largo de este constructo teórico, cabe la necesidad de señalarlos dada su operatividad en el propósito mismo de la investigación. En ese orden de ideas, se tiene la Resolución de problemas, Locus de control o atribución de Causalidad, Atribuciòn de intencionalidad (Shure \& Spivack, 1972) y toma de perspectiva (Enright \& Lapsley, 1980), las cuales presentan subhabilidades e indicadores que le caracterizan.

En consonancia con todo este soporte teórico (Shure \& Spivack, 1972; Enright \& Lapsley, 1980;Schunk y Zimmerman, 1994; Chávez y Rodríguez, 2017), abordar las interacciones sociocognitivas lleva consigo las percepciones y creencias sobre su propia autoeficacia, las cuales influyen en la forma como alcanzan sus logros de aprendizaje autorregulado, bien sea para el éxito o fracaso. De este modo, la motivación cobra una vital importancia por cuanto se concibe como la actividad intencionada, interna y sostenida.

\section{METODO}

Dado el propósito que asiste este trabajo investigativo, se adoptó un enfoque mixto (cuantitativo - cualitativo) secuencial multimétodos dado el aporte desde la estadística descriptiva (Hernández et al, 2014) y la incidencia de la investigación acción para efectos de procesos de investigación en el aula (Restrepo, 2004). Cada uno de los instrumentos aplicados (desde procesos de observación, talleres investigativo y el uso de diarios de campo y rúbricas evaluativas), a una muestra representativa no probabilística intencionada del Programa de Formación complementaria (docentes en formación inicial) de los diferentes niveles desde introductorios hasta cuarto semestre.

El proceso investigativo se resume en una fase diagnóstica (fase descriptiva) que a través del uso de softwarede análisis como el SPSS (cuantitativo) y el atlas ti (cualitativo), en correspondencia a la lectura de una matriz de referencia donde se muestra una relación horizontal entre las habilidades de pensamiento crítico y las habilidades sociocognitivas, se lora establecer un acercamiento respecto a dichas habilidades, estableciéndose un punto de partida para el diseño de una propuesta pedagógica que permita coadyuvar a cada una de las habilidades en las que se encontraron dificultades 0 vacíos.

\section{RESULTADOS Y ANÀLISIS}

\section{Identificación de habilidades de pensamiento crítico y sociocognitivas}

Dentro de los resultados concebidos se reitera desarrollo de una fase diagnóstica que se centró en la identificación de las habilidades de pensamiento crítico y habilidades sociocognitivas presentes en losdocentes en formación inicial de la Normal Superior de Sincelejo. Para ello fue determinante establecer una matriz de referencia (Ver tabla 1) que señalara las habilidades e indicadores de pensamiento crítico (Facione, 2007), desde sus niveles de complejidad y habilidades e indicadores de interaccionessociocognitivas (Shure \& Spivack, 1972; Enright \& Lapsley, 1980; Schunk y Zimmerman, 1994) manteniendo una 
relación horizontal entre ambas habilidades y sus indicadores.

Tabla 1.

Matriz de relación de habilidades de pensamiento Crítico habilidades sociocognitivas

\begin{tabular}{|c|c|c|c|}
\hline $\begin{array}{l}\text { Habilidades de } \\
\text { pensamiento } \\
\text { Crítico }\end{array}$ & $\begin{array}{c}\text { Indicadores de } \\
\text { Pensamiento Crítico: }\end{array}$ & $\begin{array}{r}\text { Habilidades } \\
\text { Sociocognitivas }\end{array}$ & Indicadores de habilidades sociocognitivas \\
\hline Interpretación & $\begin{array}{l}\text { - Decodifica los } \\
\text { elementos importantes } \\
\text { de experiencias, } \\
\text { situaciones, datos. } \\
\text { - Categoriza elementos. } \\
\text { - Clarificar el sentido. }\end{array}$ & $\begin{array}{l}\text { - La resoluciónde } \\
\text { problemas } \\
\text { interpersonales }\end{array}$ & $\begin{array}{l}\text { - Pensamiento Alternativo: Genera mentalmente } \\
\text { diferentes soluciones que pueden ser utilizadas } \\
\text { para resolver un problema en común. } \\
\text { - Pensamiento medios-fines: planea y coordina una } \\
\text { serie de pasos determinados para el logro de un } \\
\text { objetivo social, reconociendo posibles obstáculos } \\
\text { asi como la relevancia de las distintas alternativas } \\
\text { para alcanzar la meta. } \\
\text { - Pensamiento en relación a consecuencias: } \\
\text { habilidad de anticipar posibles consecuencias de un } \\
\text { acto interpersonal. } \\
\text { - Pensamiento causal: habilidad de relacionar un } \\
\text { suceso con otro en el tiempo. } \\
\text { - Sensitividad a los problemas interpersonales: } \\
\text { habilidad de reconocer situaciones } \\
\text { interpersonales conflictivas. }\end{array}$ \\
\hline Inferencias & $\begin{array}{l}\text { - Formula conjeturas e } \\
\text { hipótesis. } \\
\text { - Considera la información } \\
\text { pertinente. } \\
\text { - Saca conclusiones que } \\
\text { se desprendandedatos, } \\
\text { enunciados, creencias. } \\
\text { - Propone alternativas. } \\
\text { - Saca conclusiones. }\end{array}$ & $\begin{array}{l}\text { - Anticipación de } \\
\text { consecuencias. } \\
\text { - la atribución de } \\
\text { causalidad o } \\
\text { locus de control, la } \\
\text { atribución de } \\
\text { intencionalidad y } \\
\text { la toma de } \\
\text { perspectiva }\end{array}$ & $\begin{array}{l}\text { Generación (Resolución) del mayor número de } \\
\text { alternativas posibles, que contribuyan a la solución del } \\
\text { problema. }\end{array}$ \\
\hline Análisis & $\begin{array}{l}\text { - Examinar las ideas y las } \\
\text { descompone en sus } \\
\text { partes fundamentales. } \\
\text { - Establece relaciones que } \\
\text { le permiten hacer } \\
\text { inferencias. } \\
\text { - Analizar argumentos. }\end{array}$ & - Autoevaluación. & $\begin{array}{l}\text { - Considera, sopesa la pertinencia de susaportes en un } \\
\text { trabajo en equipo. } \\
\text { - Identifica los pasos que se siguen para reconocer un } \\
\text { problema, sus factores determinantes y posibles } \\
\text { alternativas desolución. } \\
\text { - Explica claramente la línea de razonamientoporla } \\
\text { cual llega a conclusiones. } \\
\text { - Internaliza las reglas de la comunicaciónefectiva. }\end{array}$ \\
\hline Explicación & $\begin{array}{l}\text { - Describe métodos y } \\
\text { resultados. } \\
\text { - Justifica procedimientos. } \\
\text { - Presenta argumentos } \\
\text { completos y bien } \\
\text { razonados. }\end{array}$ & - Autorregulación. & $\begin{array}{l}\text { Realiza constantes esfuerzos metacognitivos de } \\
\text { evaluación y realimentación para ajustar sus conductas y } \\
\text { estrategias. }\end{array}$ \\
\hline
\end{tabular}




\begin{tabular}{|c|c|c|c|}
\hline Autorregulación & $\begin{array}{l}\text { Autoexamen. } \\
\text { Autocorrección. }\end{array}$ & $\begin{array}{l}\text { Previsión, } \\
\text { actuación y } \\
\text { autorreflexión } \\
\text { (Zimmerman y } \\
\text { Schunk, 1989, p. } \\
\text { 53). }\end{array}$ & $\begin{array}{l}\text { - Sopesa su respuesta a partir de la intencionalidad } \\
\text { que acompaña el mensaje enviado por su } \\
\text { interlocutor. } \\
\text { - Calibra sus respuestas haciendo entonaciones y } \\
\text { aportes de acuerdo a la intención de su } \\
\text { intervención. } \\
\text { - "El sujeto tiene la capacidad de evaluar sus propios } \\
\text { objetivos de aprendizaje, de monitorear sus } \\
\text { conductas y procesos cognitivos y de utilizar los } \\
\text { resultados de esta evaluación para regular su } \\
\text { propio aprendizaje" (Zimmerman, 1990, 2015; } \\
\text { Pintrich, 2004;), citados en Chávez y Rodríguez } \\
\text { (2017, p. 53). }\end{array}$ \\
\hline Evaluación & $\begin{array}{l}\text { Evaluar afirmaciones y } \\
\text { argumentos. }\end{array}$ & $\begin{array}{l}\text { Toma de perspectiva } \\
\text { (Cognitiva-Social) }\end{array}$ & $\begin{array}{l}\text { Tiene la habilidad cognitiva y emocional para } \\
\text { interpretar los pensamientos y emociones de } \\
\text { losotros y actuar en consecuencia. (Enright } \\
\text { \&Lapsley,1980). }\end{array}$ \\
\hline
\end{tabular}

Fuente: Facione (2007); Shure \& Spivack, (1972); Enright \& Lapsley, (1980); Schunk y Zimmerman, (1994).

Teniendo en cuenta todos estos elementos como punto de partida de análisis diagnóstico, se logra constatar para efectos de las habilidades de pensamiento crítico, valoraciones en tres de sus niveles: interpretación, inferencias y análisis. En ese orden, para la habilidad de interpretación, la cual según Facione (2007) implica comprender y expresar el significado o la relevancia de una amplia variedad de experiencias, situaciones, datos, etc. En ese sentido, el comportamiento de los datos procesados concibe un desempeño preocupante en este nivel, de manera que, sus valoraciones porcentuales señalan un $60 \%$ para bajo, lo que implica que los docentes en formación inicial, "hace uso de lo literal o de la textualidad para identificar algunos elementos de una amplia variedad de experiencias, situaciones, datos, etc" Seguidamente se constata un 30\% en básico y sólo un $10 \%$ en alto, es decir, solo una parte poco significativa de los estudiantes del Programa de Formación Complementaria (PFC) realmente "decodifica los elementos más importantes de una amplia variedad de experiencias, situaciones, datos, etc; categorizándolos y dándole sentido lógico entre ellos, reconociendo además, patrones que le permitan identificar los elementos de dichas situaciones" (ver figura 1).

En definitiva, el comportamiento de la figura anterior, se está manifestando una lectura literal para identificar e interpretar la variedad de elementos a los que se ven los docentes en formación inicial expuestos, por lo que su comprensión es fragmentada y aislada, concibiéndose ciertas dificultades para mostrar la coherencia con lo que pretenden interpretar, denotándose además, dificultades para establecer las relaciones que se dan entre los elementos que identifican en las experiencias, situaciones y datos. 


\section{Figura 1.}

Valoración de la habilidad de pensamiento crítico en interpretación.

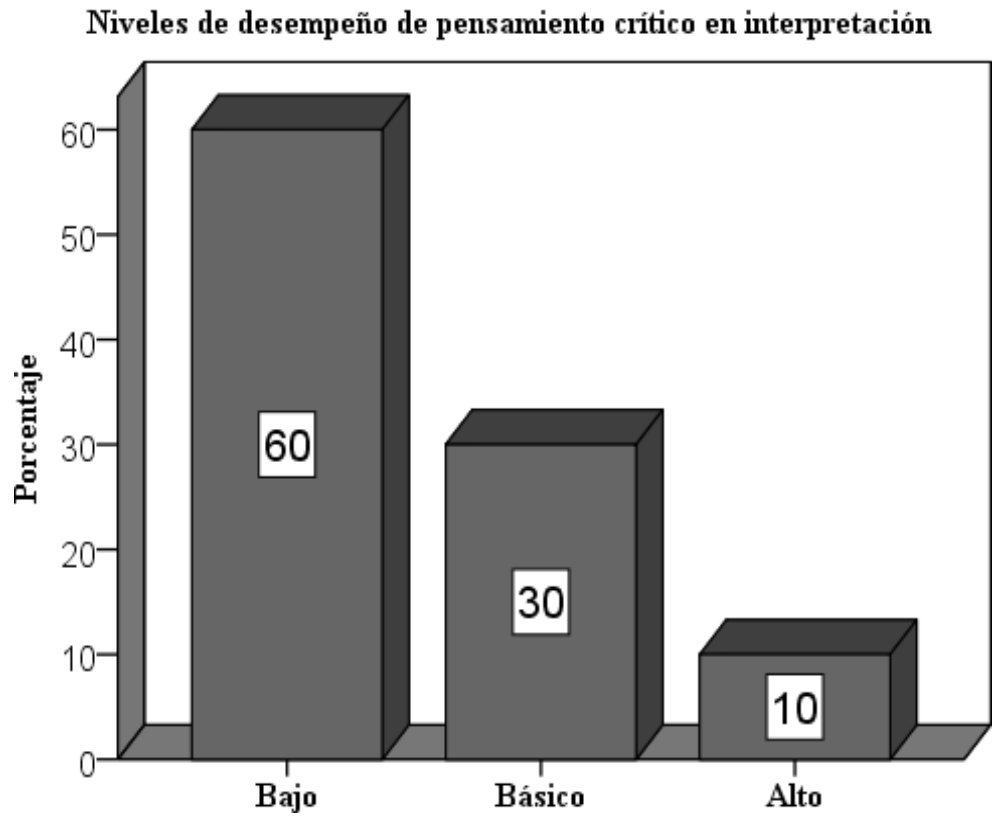

Fuente: elaboración propia.

Por su parte, en la habilidad de análisis, consistente en la identificación de las relaciones de inferencia reales y supuestas entre enunciados, preguntas, conceptos, etc, con propósitos de expresar ideas, creencias u opiniones (Facione, 2007). Se encontró que el 80\% de los docentes en formación inicial, presentan un desempeño bajo, lo que implica en estos "dificultades en la descomposición de la idea en sus partes, por lo que le es casi imposible establecer relaciones entre ellas; por lo que es incapaz de identificar patrones que le permitan inferir causas y efectos dentro de esos pensamientos.

Finalmente un 20\% con desempeño básico, es decir, sólo esta porción de la muestra representativa "Examina las ideas y las descompone en sus partes fundamentales, para establecer relaciones que le permita hacer inferencias que explican el porqué, cómo y cuándo de ellas. Sin embargo, tiene dificultades para determinar y comprender el principio que integra dicha idea. De igual manera, aunque los docentes en formación inicial identifiquen algunos patrones que le permiten inferir causas y efectos dentro de esos pensamientos, estos adolecen de la construcción de un modelo mental que le facilite la comprensión de dichas ideas" (Ver figura 2). 


\section{Figura 2.}

Valoración de la habilidad de pensamiento crítico en análisis.

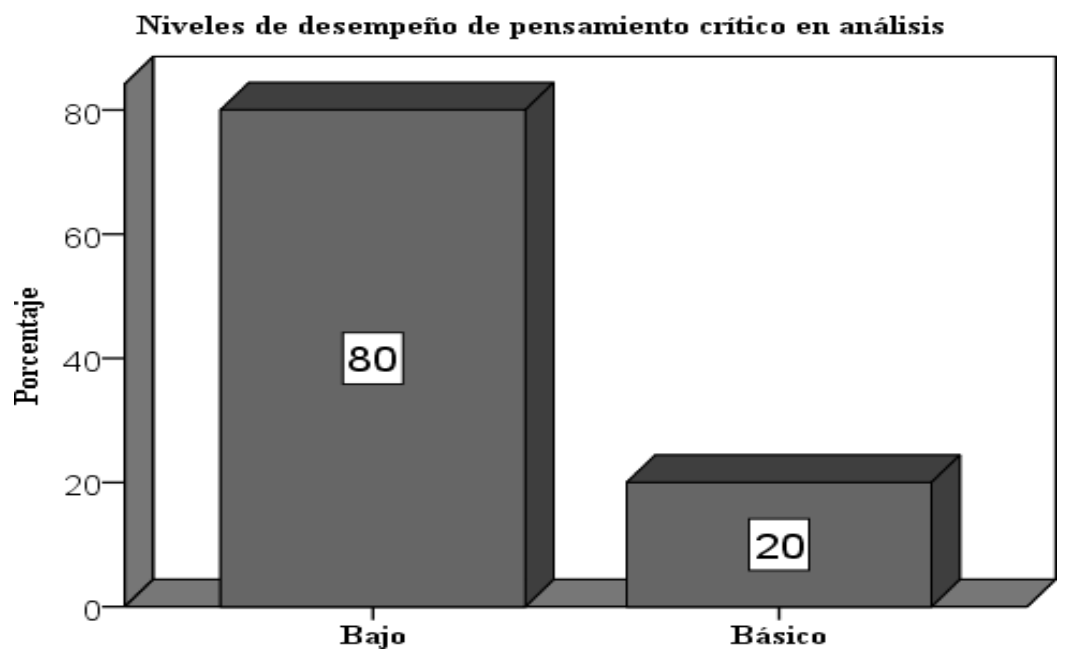

Fuente: elaboración propia.

Finalmente en la habilidad inferencia, siendo la capacidad de identificar y asegurar los elementos necesarios para sacar conclusiones razonables; formularconjeturas e hipótesis; considerar la información pertinente para llegar a consecuencias lógicas (Facione, 2007), se pudo constatar un 90\% de desempeño bajo, frente a un 10\% básico. Para el primer desempeño se concibe que los docentes en formación inicial, "identifican algunos datos, enunciados, principios, evidencias, juicios, creencias,opiniones, conceptos y descripciones, y se le dificultaestablecer relaciones entre estos. Asimismo, estos pueden elaborar preguntas simples sobre los datos expuestos. Sin embargo, la elaboración de conjeturase hipótesis queda limitada a lo que logra comprender". (Ver figura 3).

\section{Figura 3.}

Valoración de la habilidad de pensamiento crítico en inferencia.

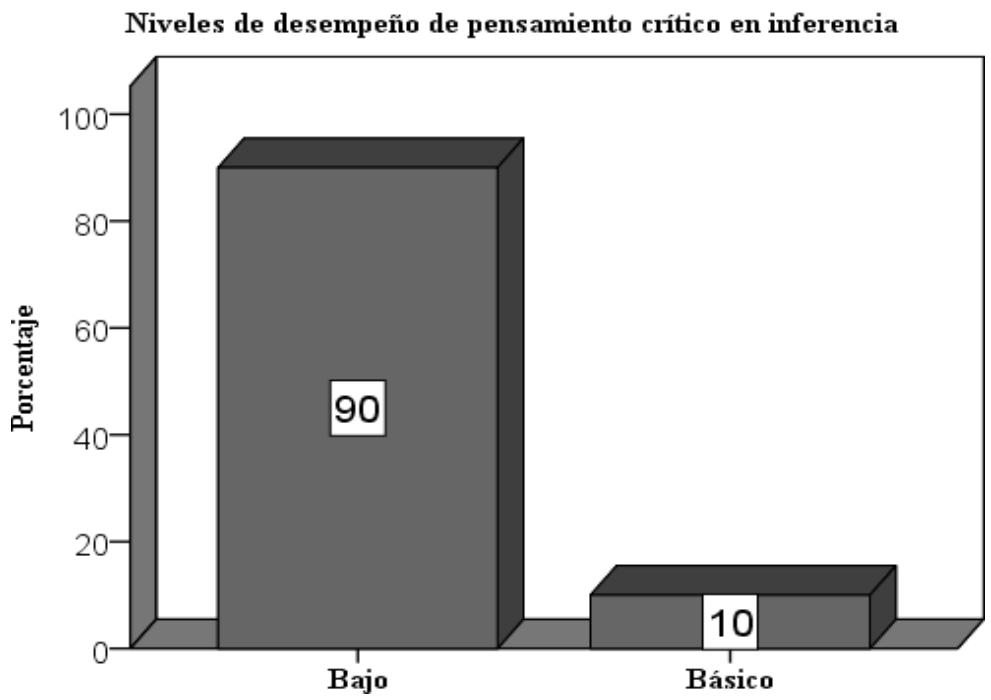

Fuente: elaboración propia. 
Cabe mencionar que, sólo una pequeña parte de la muestra puede "Identificar datos, enunciados,principios, evidencias, juicios, creencias, opiniones, conceptos y descripciones que le permitan establecer relaciones de causa - consecuencia, considerándolas según su pertinencia; y al tiempo, pueden elaborar preguntas sobre estos datos identificados con el propósito de sacar conclusiones que ayuden a comprender el pensamiento expuesto, en definitiva, no logran extraer la información pertinente para llegar a consecuencias lógicas

Ahora bien, en consonancia con los resultados concebidos desde la interpretación, análisis e inferencia, en orden de gradualidad, es notable considerar desde su lógica, que si se presentan desempeños bajos en la interpretación, por ende se verán reflejados en dificultades para realizar análisis en las partes fundamentales de un texto, por lo tanto, al momento de establecer relaciones entre ellas también serán complejas, por las evidentes dificultades para inferir causas y efecto dentro de esos pensamientos, adoleciendo a una construcción y formulación de posibles hipótesis y conjeturas que lo direcciones a conclusiones pertinentes.

De manera generalizada, las relaciones en cada una de las habilidades anteriormente señaladas dejan en evidencia en cuanto a los docentes en formación inicial, que estos presentan poco procesamiento de la información, dificultades en la construcción de significados, limitaciones en la competencia semántica, comprensión de textos en niveles literales, en definitiva una progresión temática poco cohesiva y coherente (ver figura 4). Cuyas dificultades se reflejan además en las interacciones sociocognitivas, por cuanto el pensamiento crítico se concibe como un proceso metadiscursivo que se enriquece en las interacciones sociocognitivas a través de la práctica comunicativa donde cobran cabida los argumentos y contraargumentos de los interlocutores.

Cuando se habla sobre una persona que tiene el pensamiento crítico estamos hablando de un pensamiento no como algo estático, sino como una habilidad que tiene ciertos componentes que involucra esos factores cognitivos en la interacción que tenemos con nosotros. De este modo, al hablar desde la interacción sociocognitiva, desarrollamos la habilidad para conjugar la información que estamos recibiendo de nuestro interlocutor para analizarla, para evaluarla y para interpretarla, y en ese sentido, retroalimentarla y enviar esa información de retorno que son las respuestas quenosotros damos al tener un interlocutor válido, es decir, el sentido y el significado que se le dé a la palabra debe considerarun contexto determinado que excluye la subjetividad y excluye las creencias particulares de cada quién porque está llevarían a generar sesgos en la interpretación de la información. 


\section{Figura 4.}

Relación de criterios asociados de pensamiento crítico con las interacciones sociocognitivas.

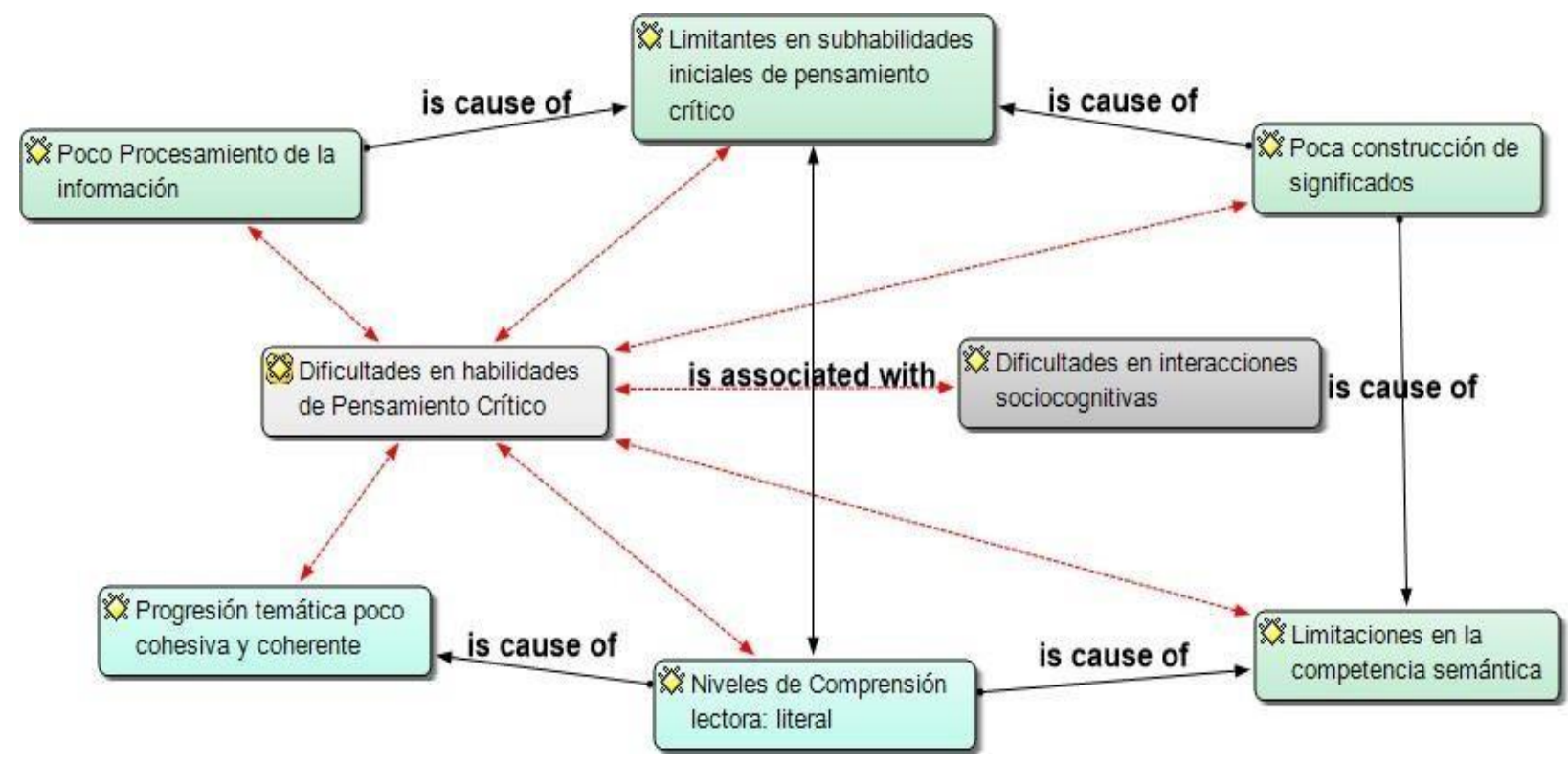

Fuente: elaboración propia.

A raíz de estas determinaciones, cabe la necesidad de llevar a cabo procesos de intervención que partan de un ejercicio interdisciplinar que permita la movilización de estrategias que potencien habilidades de pensamiento crítico, y dada su influencia en las habilidades sociocognitivas, que le involucre al docente en formación inicial la estimulación de un pensamiento alternativo que donde pueda generar posibles soluciones ante las problemáticas que el contexto demanda desde su ejercicio práctico dentro y fuera del aula., o en su defecto anticiparse a situaciones problémicas, pero más importante aún es que el docente en formación inicial pueda realizar constantes ejercicios metacognitivos, que planifique, supervise y evalúeprocesos para ajustar conductas y estrategias, o en definitiva presente procesos de autorregulación, en el que de acuerdo con Zimmerman (1990, citado en Chávez \& Rodríguez (2017, p. 53), el sujeto tiene la capacidad de evaluar sus propios objetivos de aprendizaje, de monitorear sus conductas y procesos cognitivos y de utilizar los resultados de esta evaluación para regular su propio aprendizaje" .

Desde esa óptica, una mirada de intervención pedagógica desde el ejercicio mismo de la interdisciplinariedad en el programa de formación complementaria, correspondería al involucramiento devarias fases: una fase de conceptualización, otra de acercamiento a la practicidad de estrategias problematizadoras y por último un ejercicio secuencial de aplicación de las estrategias problematizadoras donde se evidencie un cruce entre las habilidades de pensamiento crítico y habilidades sociocognitivas (ver figura 5). 
Figura 5. Relación de fases de intervención pedagógica para el pensamiento crítico y habilidades sociocognitivas.

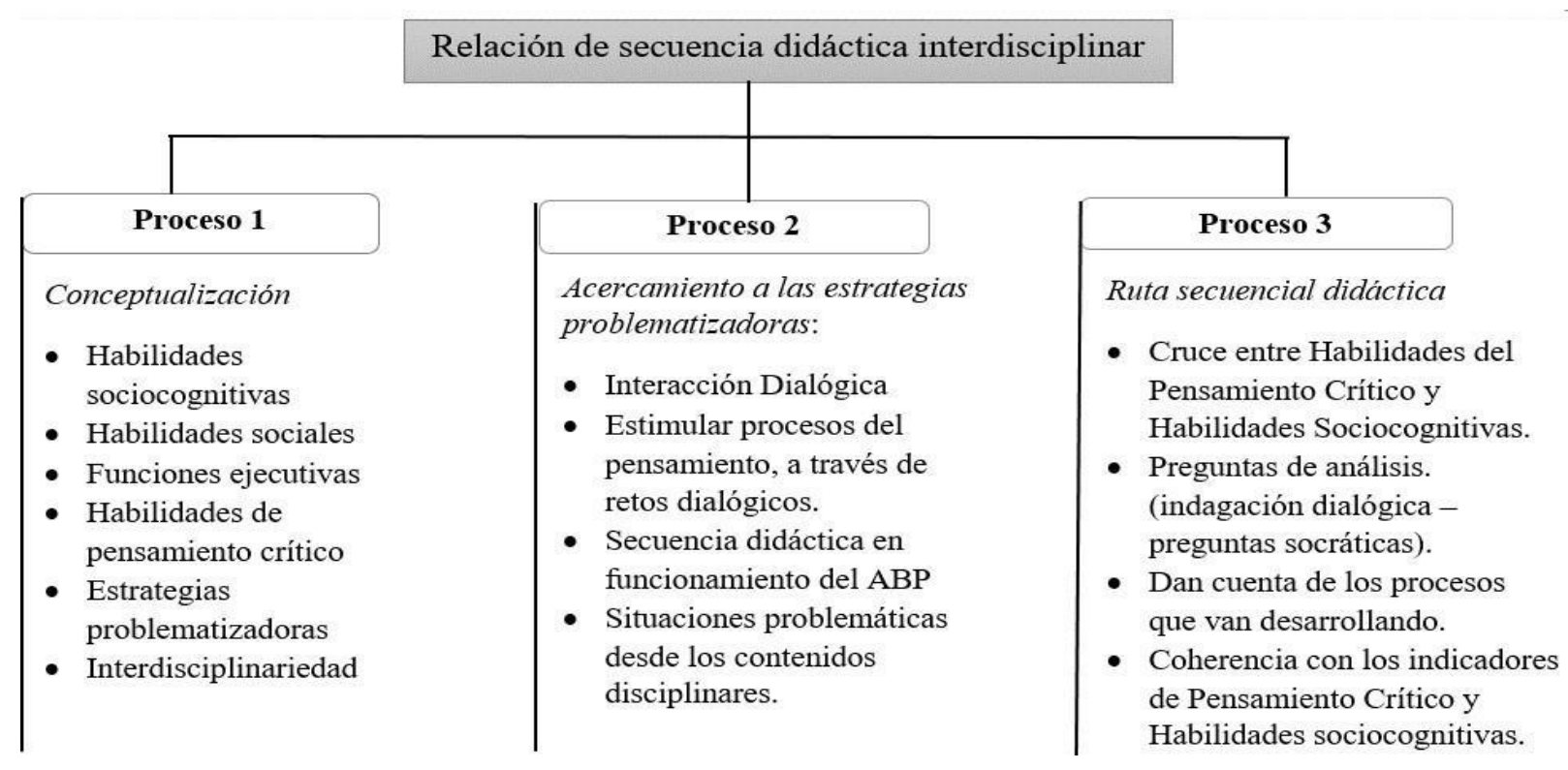

Fuente: elaboración propia.

Como se puede percibir, la primera fase (conceptualización), se es necesario desde guías interdisciplinarias, dar a conocer y ejercitar los conceptos básicos que se van a estar utilizando durante el proceso de intervención, darrespuesta al cómo son las interacciones sociocognitivas, las habilidades sociales, habilidades sociocognitivas, habilidades de pensamiento crítico. Seguidamente una segunda fase que responda al acercamiento de las estrategias problematizadoras que permitan estimular dichas habilidades. El trabajo se centra en una forma de entrar en confianza con el manejo de las estrategias problematizadoras desde el uso de estrategias dialógicas (Fisher, R. 2013) permitiendo que los docentes en formación inicial, desarrollen la comprensión entre ellos a partir de preguntas dialógicas. En este punto, estos pueden expresar su parecer desde su cotidianidad, desde su punto de vista, entrando en confianza para manejar sus sentimientos y emociones y poder seguir avanzando en algunos aspectos claves que tengan que ver con las habilidades cognitivas y procesos complejos.

De igual manera este entrenamiento (uso de estrategias problematizadoras) permite concebir al aprendizaje basado en problemas (ABP), e integre los elementos que forman al ABP, y estimule a los educandos respecto a los procesos de pensamiento, pero considerando además una ruta sobre la ejercitación que hacen desarrollando las guías interdisciplinares que se plantean. No obstante, dentro del proceso de aplicabilidad del ABP, también se hayan inherentes unos retos dialógicos que son los que nos permiten desarrollar el pensamiento ante situaciones problémicas (Escribanoy Del Valle, 2010), en los cuales ellos puedan dar respuesta a construcciones ya de una manera más compleja en donde vayan involucrando los contenidos disciplinares que se hayan venido adquiriendo. 
Finalmente en una tercera fase que es precisamente donde se concibe una secuencialidad didáctica, es decir, una ruta metodológica para poder implementar y fomentar el pensamiento crítico en los docentes en formación inicial, haciéndose un cruce entre estas habilidades de pensamiento crítico y la habilidades de pensamiento, habilidades sociocognitivas, en un tipo de preguntas de indagación, o preguntas socráticas que estimulen el análisis de los docentes en formación inicial y comprometan en ellos al pensamiento para que den cuenta de cada uno de esos procesos que ellos llevan a cabo estas preguntas están contempladas dentro de las categorías que nos habla el pensamiento crítico de Facione, P. (2007) Entonces en este seguimiento de las preguntas se va entrando al proceso de la complejidad del pensamiento

\section{CONCLUSIONES}

A la luz de los instrumentos aplicados y los resultados constatados, que dio lugar a la identificación de niveles de desempeño en habilidades de pensamiento crítico preocupantes dado su bajos niveles concebidos, se concluye que, existe una estrecha relación de gradualidad respecto a la complejidad de estas habilidades, mostrando pocas habilidadesde pensamiento que conduzcan a realizar análisis e inferencias, por lo que su literalidad en el ejercicio mismo de la interpretación es más pronunciado en los docentes en formación inicial.

El pensamiento crítico está presente en interacciones sociocognitivas a través de los diversos niveles de complejidad, de las acciones cognitivas que le caracterizan desde el desarrollo del razonamiento, el juicio, en la metacognición, además de ello, nos permite que en esa interacción con los otros, se pueda realizar acciones motivacionales y de autorregulación, con la mirada de poder mantener el esfuerzo cognitivo y mantener ese proceso del pensamiento crítico, en la medida que, el sujeto que cuestionamos, evaluamos y hacemos los procesos inferenciales pueda sentar un diálogo o un debate a la altura del rigor que implica la claridad y la precisión y la exactitud del pensamiento crítico.

Desde esa postura, se concluye la pertinencia de favorecer los procesos desde la apropiación teórico-práctica de las interacciones sociocognitivas, pensamiento crítico, y estrategias problematizadoras que permitan establecer conflictos cognitivos en los docentes en formación inicial, para que ante el proceso de intervención se pueda tener una dinámica de enseñanza-aprendizaje reflexiva y crítica. Para lo cual, se recomienda adoptar un proceso evaluativo que demande acciones metacognitiva que lleven a la finalidad de un aprendizaje autorregulado.

\section{REFERENCIAS BIBLIOGRÁFICAS}

Azevedo, R. (2007). Understanding the complex nature of self-regulatory processes in learning with computer-based learning environments: an introduction [Comprender la naturaleza compleja de los procesos de autorregulación en el aprendizaje con entornos de aprendizaje basados en computadoras: una introducción. MetacognitionLearning, 2, 57-65.

Bandura, A. (1997). Self-efficacy: The exercise of control [Autoeficacia: El ejercicio del control]. New York: Freeman.

Chávez, B., \& Rodríguez, M. (2017). Aprendizaje autorregulado en la teoría sociocognitiva: Marco conceptual y posibleslíneas de investigación. Ensayos Pedagógicos, 12(2), 47-71. 
Enright, R. \& Lapsley, D . ( 1980). Social role-taking: a review of thc construcls, measures and measurcments properties. eviewifEdltcalionalRe earch. 50,647-674.

Escribano, \& Valle, y. D. (2010). El Aprendizaje Basado en Problemas. Una propuesta Metodologica enEducaciónSuperior. Madrid: Narcea.

Facione, P. (2007). Pensamiento Crítico:¿ Qué es y por qué es importante. Insight assessment, 22.

Farfas, K, C. F., Grothusen, S., Muñoz, M. J., \& Von Freeden, P. (2006). Revisión de las habilidades socio- cognitivas en la infancia temprana. Summa psicológica UST, 3(1), 31-42.

Fisher, R. (2013). El diálogo creativo en el aula. Ediciones Morata.

Hernández, R., Fernández, C., \& Baptista, P. (2014). Metodología de la investigación McGraw-Hill. México DF.

Restrepo Gómez, B. (2004). La investigación-acción educativa y la construcción de saber pedagógico.

Educación y Educadores, (7), 45-55.

Rinn, R. \& Markle,A. ( 1979). Modification ofsocial skills deficits in children. En A. Bellack y M. Hersen (Eds.) Rereorc/1aud proclice lit J. ocial slalls hmiuilg, New York: Plenum Press.

Schunk, D. H. (2004). Learning theories: An educational perspective (4th ed.) [Teorías del aprendizaje: una perspectiva educativa (4th ed.)]. Upper Saddle River: Pearson Education.

Schunk, D. H. y Zimmerman, B. J. (Eds.). (1994). Self-regulation of learning and performance[Autorregulación del aprendizaje y el rendimiento]. Hillsdale: Lawrence Erlbaum.

Shure, M. \& Spivack, G. (1972). Means-ends thinking, adjustment and social class among clcmentary-schoolagcdchildrcn. Joumal of Comulltitg rmd Clinical Psyc!JO!Og)', 38, 248-353.

Shure, M. \& Spivack, G. ( 1978). Problem So/Jiillg Techniques in Chilrlreanilg. London: Josscy-Bass Pub. Vélez G. (2013). Una reflexión interdisciplinar sobre el pensamiento crítico. Revista Latinoamericana de Estudios Educativos (Colombia), 9(2), 11-39.

Zimmerman, B. J. (2008). Investigating self-regulation and motivation: Historical background, methodological development, and future prospects [Investigación de la autorregulación y motivación: Antecedentes históricos, desarrollo metodológico y perspectivas futuras]. American Educational Research Journal, 45(1), 166-183.

Zimmerman, B. J. (2015). Self-Regulated Learning: Theories, Measures, and Outcomes [Aprendizaje auto- regulado: teorías, medidas y resultados]. In J. D. Wright (Ed.), International Encyclopedia of theSocial \& Behavioral Sciences (pp. 541546). Oxford: Elsevier. doi:10.1016/B978-0-08-097086- 8.26060-1 